\title{
SOLUTION OF THE DIRICHLET PROBLEM BY INTERPOLATING HARMONIC POLYNOMIALS ${ }^{1}$
}

\author{
BY J. H. CURTISS
}

Communicated by John W. Green, February 23, 1962

1. Introduction. Let $D$ be a bounded simply connected region of the complex $z$-plane which is regular for the Dirichlet problem, let $C$ be the boundary of $D$, and let $u$ be a continuous function on $C$ to the real numbers. Some time ago J. L. Walsh [1, p. 517] suggested that it might be possible to define a sequence of harmonic polynomials by coincidence with the values of $u$ in points so chosen on $C$ that the polynomials converge on $D$ to the solution of the corresponding Dirichlet problem. He showed that a convergent interpolation process of this type is available if $D$ is a circular disk $[1 ; 2]$. Recently the author [3] raised the general question anew, and Walsh [4] then extended his earlier result to elliptical disks. For both theoretical and practical reasons it seems worthwhile to develop a general convergence theory, and progress toward this goal is announced here in Theorems 3.2 and 3.3 below.

2. Existence and structure of harmonic interpolation polynomials. A harmonic polynomial of degree $n$ can be written as $h(z)=a_{0}$ $+\sum_{j=1}^{n}\left(a_{j} z^{j}+\bar{a}_{j} \bar{z}^{j}\right)$, where $a_{0}$ is real, $a_{n} \neq 0$, and the bar over a letter denotes conjugate complex. The real and imaginary parts of any finite complex linear combination of the monomials $1, z, z^{2}, \cdots$, $\vec{z}, \bar{z}^{2}, \cdots$ are harmonic polynomials. Therefore if such a combination vanishes identically on the boundary of a bounded region, then all of the coefficients must be zero. If $p_{1}(z), p_{2}(z), \cdots, p_{n}(z)$ are any complex polynomials in $z$ of respective degrees $1,2, \cdots, n$, then the expression $b_{0}+\sum_{j=1}^{n}\left[b_{j} p_{j}(z)+\bar{b}_{j}\left(p_{j}(z)\right)^{-}\right]$is also a harmonic polynomial of degree $n$, where now $\left(p_{j}(z)\right)^{-}$denotes the complex conjugate of $p_{j}(z)$.

Given points $z_{1}, z_{2}, \cdots, z_{2 n+1}$ and real or complex numbers $u_{1}, u_{2}, \cdots, u_{2 n+1}$, a necessary and sufficient condition that there exists a unique set of $2 n+1$ numbers $c_{0}, c_{1}, \cdots, c_{n}, d_{1}, \cdots, d_{n}$ satisfying the system of linear algebraic equations

$P\left(z_{h}\right)=c_{0}+\sum_{j=1}^{n}\left[c_{j} p_{j}\left(z_{h}\right)+d_{j}\left(p_{j}\left(z_{h}\right)\right)^{-}\right]=u_{h}, \quad h=1,2, \cdots, 2 n+1$,

1 This research was supported in part by the United States Air Force through the Air Force Research and Development Command, under Contract Number AF 49(638)-862. 
for each of the possible assignments of the numbers $u_{h}$ is that the matrix

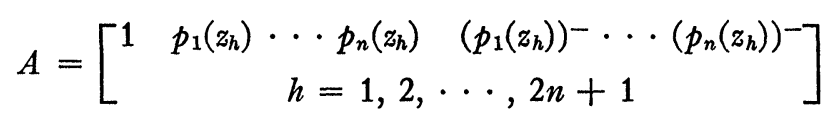

is non-singular. This non-singularity condition, henceforth here to be called the $n$-s condition, is equivalent (see [3]) to the condition that the points $z_{1}, z_{2}, \cdots, z_{2 n+1}$ shall be distinct and shall not all lie on an algebraic curve with an equation of the type $H(z)=0$, where $H(z)$ is a harmonic polynomial of degree at most $n$. If the n-s condition obtains, and if the numbers $u_{h}$ are all real, so that $P\left(z_{h}\right)=\left(P\left(z_{h}\right)\right)^{-}$, then $d_{j}=\bar{c}_{j}$, so $P(z)$ is a harmonic polynomial. It is the "unique" harmonic polynomial of degree at most $n$ which takes on the values $u_{h}$ at the points $z_{h}, h=1,2, \cdots, 2 n+1$, with uniqueness to be understood in the sense that for a given choice of the base polynomials $p_{1}(z), \cdots$, $p_{n}(z)$, their coefficients in $P(z)$ are uniquely determined. If two harmonic polynomials, each of degree at most $n$, coincide in $2 n+1$ points satisfying the $n$-s condition, then the two polynomials are identically equal for all $z$.

Consider now the matrix $A_{k}(z)$ obtained by replacing the $k$ th row in $A$ by the row $1, p_{1}(z), \cdots, p_{n}(z),\left(p_{1}(z)\right)^{-}, \cdots,\left(p_{n}(z)\right)^{-}$. We assume that the $\mathrm{n}$-s condition is in effect. The expression

$$
H_{n}(z)=\sum_{k=1}^{2 n+1} u_{k} \frac{\operatorname{det} A_{k}(z)}{\operatorname{det} A}
$$

is a linear combination of $1, p_{1}(z), \cdots, p_{n}(z),\left(p_{1}(z)\right)^{-}, \cdots,\left(p^{n}(z)\right)^{-}$, such that $H_{n}\left(z_{h}\right)=u_{h}, h=1, \cdots, 2 n+1$. Therefore when the numbers $u_{h}$ are real, this is the unique harmonic polynomial of degree at most $n$ which behaves in this way.

It is important to note that given any bounded region, for any positive integer $n$ there always exists at least one set of $2 n+1$ points on the boundary $C$ of the region which satisfy the $n$-s condition. To show this, it suffices to work with the specialization of $A$ in which $p_{j}(z)=z^{j}$, $j=1, \cdots, n$. Certainly the first $n+1$ points can be chosen on $C$ so that the upper left hand $(n+1)$-rowed principal minor of $A$ is nonsingular, because this minor is merely a Vandermonde matrix. The determinant of the upper left hand $(n+2)$-rowed principal minor is a linear combination of the monomials $1, z_{n+2}, \cdots, z_{n+2}^{n}, \bar{z}_{n+2}$, and if it vanished for all $z_{n+2}$ on $C$ then all of the coefficients in this linear combination would have to be zero, which is not so. It is obvious how to complete the proof by induction. 
3. Convergence theorems. We shall now be considering an infinite sequence of harmonic polynomials $H_{1}(u ; z), H_{2}(u ; z), \cdots, \cdots$, found by interpolation to the values of a function $u$ given on the boundary $C$ of a bounded open point set $D$. The polynomial $H_{n}(u ; z)$ is of degree at most $n$ and coincides with the values of $u$ in a point set on $C$ which we shall denote by $S_{n}=\left\{z_{n 1}, z_{n 2}, \cdots, z_{n, 2 n+1}\right\}$. For each $n$ it is assumed that $S_{n}$ satisfies the n-s condition. We write $H_{n}(u ; z)$ in the form (2.1):

$$
H_{n}(u ; z)=\sum_{k=1}^{2 n+1} u\left(z_{n k}\right) \frac{\operatorname{det} A_{n k}(z)}{\operatorname{det} A_{n}},
$$

where $A_{n}$ and $A_{n k}(z)$ denote respectively the matrices $A$ and $A_{k}(z)$ of $\S 2$ when they are set up using the interpolation points $S_{n}$. We let

$$
T_{n}(z)=\sum_{k=1}^{2 n+1}\left|\frac{\operatorname{det} A_{n k}(z)}{\operatorname{det} A_{n}}\right| .
$$

For any fixed $z$ and $n, H_{n}(u ; z)$ is a linear functional on the space of functions $u$, and $T_{n}(z)$ is its norm.

THEOREM 3.1. Let $\Gamma$ be any compact point set which contains the boundary of some bounded region. There exists a sequence of subsets $S_{1}^{*}, S_{2}^{*}, \cdots$ of $\Gamma$, each satisfying the $n$-s condition, such that $T_{n}(z)$ $\leqq 2 n+1, n=1,2, \cdots, z$, on $\Gamma$, when $T_{n}(z)$ is constructed using $S_{n}{ }^{*}$.

The condition that there shall be the boundary of a bounded region somewhere in $\Gamma$ is inserted merely to insure that for each $n$, some subset $S_{n}$ of $\Gamma$ exists which satisfies the n-s condition. (See $\$ 2$ above.) The determinant of $A_{n}$ is a continuous function of the $2 n+1$ independent variables $z_{1}, z_{2}, \cdots, z_{2 n+1}$. Therefore $\left|\operatorname{det} A_{n}\right|$ has a maximum on $\Gamma$ which is positive (n-s condition). If $S_{n}{ }^{*}$ denotes a maximizing point set and $A_{n}$ and $A_{n k}(z)$ are constructed using $S_{n}^{*}$, then $\left|\operatorname{det} A_{n k}(z)\right| \leqq\left|\operatorname{det} A_{n}\right|$ for all $z$ on $\Gamma$, and the result follows from this.

It is possible to show by using the Hadamard determinant inequality that when $\Gamma$ is a circle the points $S_{n}{ }^{*}$ must be equally spaced on it.

THEOREM 3.2. Let $D$ be a point set consisting of a finite number of mutually exterior bounded simply connected regions. Let $u$ be a function which is continuous on $C$, the boundary of $D$. If there exist harmonic polynomials $h_{1}(z), h_{2}(z), \cdots$ of respective degrees at most $1,2, \cdots$ such that $u(z)-h_{n}(z)=o(1 / n)$ uniformly on $C$, then there exists $a$ sequence of interpolation point sets $S_{1}, S_{2}, \cdots$ on $C$, each satisfying the $n$-s condition, and such that $\lim _{n \rightarrow \infty} H_{n}(u ; z)$ exists uniformly on $D+C$ 
and is equal to the solution of the Dirichlet problem for $u$ on each component of $D$.

As the sequence $S_{1}, S_{2}, \cdots$ we use the sequence which appears in Theorem 3.1. The hypothesis of Theorem 3.2 implies that there is a solution of the Dirichlet problem with boundary values given by $u$ for each component of $D$. If $U_{m}$ denotes the solution for the $m$ th component of $D$, then since $H_{n}\left(h_{n} ; z\right) \equiv h_{n}(z)$, it follows that

$$
\begin{aligned}
& \left|H_{n}(u ; z)-U_{m}(z)\right| \\
& \quad \equiv\left|\sum_{k=1}^{2 n+1}\left[u\left(z_{n k}\right)-h_{n}\left(z_{n k}\right)\right] \frac{\operatorname{det} A_{n k}(z)}{\operatorname{det} A_{n}}+h_{n}(z)-U_{m}(z)\right| \\
& \quad \leqq(2 n+2) \max _{t \in C}\left|u(t)-h_{n}(t)\right|
\end{aligned}
$$

for all $z$ on the boundary of the $m$ th component of $D$ and therefore (maximum principle) for all $z$ on the closure of this component of $D$. The theorem follows immediately.

And so we have referred the convergence problem of harmonic polynomial interpolation back to the possibility of any type of harmonic polynomial approximation with the uniform norm. Various results on the latter problem are available, many of which are associated with Walsh and his pupils. We present a typical example of the theorems which can be derived in this way.

THEOREM 3.3. Let $D$ be a region bounded by a Jordan curve $C$ of Sewell's Type W [5, p. 196]. Let the function $u$ be given on $C$ and let its first derivative $u^{\prime}$ (taken in a one-dimensional sense on $C$ ) satisfy on $C$ a Lipschitz condition of some order $\alpha, 0<\alpha<1$. Then there exists a sequence of interpolation point sets $S_{1}, S_{2}, \cdots$ on $C$ such that $\lim _{n \rightarrow \infty} H_{n}(u ; z)$ exists uniformly on $D+C$ and equals the solution of the Dirichlet problem for $u$ and $D$.

The proof consists mainly in showing that certain results of Sewell [5] on complex polynomial approximation can be carried over to harmonic polynomials. Alternatively some similar results of Mergelyan $[6$, p. 84] can be used, which permit $C$ to have corners if $u$ is smooth enough. The theorem remains true when $D$ consists of a finite number of mutually exterior Jordan regions, because Theorem 3.1 and the harmonic approximations used for comparison remain valid for this case.

Similarly it can be shown by referring to [7] that if $U$ is harmonic on a closed arbitrary Jordan region and $H_{n}(U ; z)$ interpolates on the 
boundary to $U$ in the points of Theorem 3.1 , then $H_{n}(U ; z)$ converges uniformly at a geometric rate to $U(z)$ in the closed region.

\section{REFERENCES}

1. J. L. Walsh, The approximation of harmonic functions by harmonic polynomials and by harmonic rational functions, Bull. Amer. Math. Soc. 35 (1929), 499-544.

2. - On interpolation to harmonic functions by harmonic polynomials, Proc. Nat. Acad. Sci. U.S.A. 18 (1932), 514-517.

3. J. H. Curtiss, Interpolation with harmonic and complex polynomials to boundary values, J. Math. Mech. 9 (1960), 167-192.

4. J. L. Walsh, Solution of the Dirichlet problem for the ellipse by interpolating harmonic polynomials, J. Math. Mech. 9 (1960), 193-196.

5. W. E. Sewell, Degree of approximation to a continuous function on a non-analytic curve, Proc. Nat. Acad. Sci. U.S.A. 47 (1961), 195-202.

6. S. N. Mergelyan, Uniform approximations to functions of a complex variable, Uspehi Mat. Nauk (N.S.), 7 (1952), no. 2 (48), 31-122. Amer. Math. Soc. Transl. No. 101 (1954).

7. J. L. Walsh, Maximal convergence of sequences of harmonic polynomials, Ann. of Math. 38 (1937), 321-354.

UNIVERSITY OF MiAMI 